\title{
Sonographic gastric volume and risk factors for full stomach in emergency surgery: an observational study
}

Delamarre L., Brunel E., Conil J.-M., Fourcade O., Minville V.

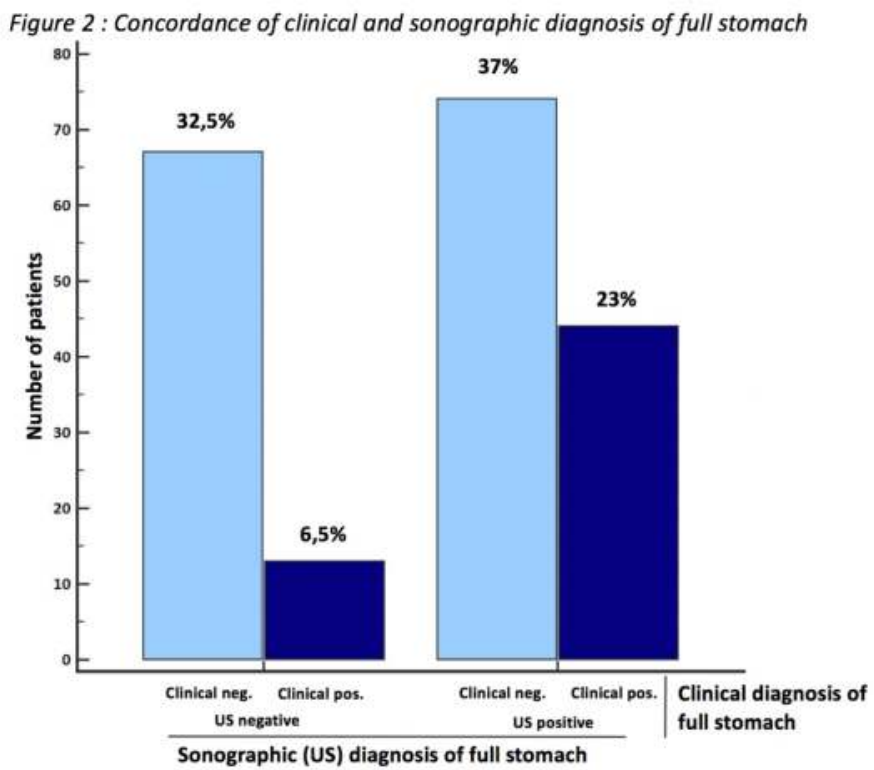

Background and Goal of Study: Perioperative pulmonary aspiration remains a life-threatening complication of emergency anesthesia. Clinical judgment based on risk factors for full stomach drives the choice of rapid sequence induction (RSI). Gastric ultrasounds (US) look promising. The primary goal was to investigate the relationship between clinical risk factors and US gastric volume. Secondary goals were concordance of clinical and US judgments and characterization of patients with undetected full stomach.

Materials and Methods: A prospective observational study was conducted at our University Hospital. Clinical judgment regarding the state of full stomach and 15 risk factors were collected, followed by US measurements of antral cross section area (CSA). A CSA $\geq 3.5 \mathrm{~cm} 2$ defined a full stomach. Concordance between judgments was tested by Cohen's Kappa.

Results and Discussion: 210 patients were included. No relationship between clinical risk factors and US full stomach was found, except for clinical ileus $(p=0.03)$. Concordance between clinical and US judgments was mediocre (Cohen's Kappa: 0.19). Patients with clinically undetected full stomach represented $37 \%$ of our cohort, but exhibited no specific risk factor but chronic kidney disease. They benefited from an adequate anesthesia (RSI or regional anesthesia) in only $70 \%$ of the cases.
Table 1 : Patients' characteristics considering sonographic full or empty stomach.

\begin{tabular}{|c|c|c|c|}
\hline & $\begin{array}{l}\text { Sonographic full stomach } \\
\qquad(\mathrm{n}=119)\end{array}$ & $\begin{array}{l}\text { Sonographic empty stomach } \\
\qquad(n=81)\end{array}$ & $p$ \\
\hline & $n$ (\%) or Median [1 195$]$ & $n(\%)$ or Median [c195] & \\
\hline \multicolumn{4}{|l|}{ Demographic data } \\
\hline Age & 50[39.3-54.7] $\mathrm{C}$ & $47(34-55]$ & 0.26 \\
\hline $\begin{array}{l}\text { BM! (kg/m2) } \\
\text { Gender }\end{array}$ & $23.7[22.9-24.6]$ & $23.2[22.5-24]$ & $\begin{array}{l}0.56 \\
0.89\end{array}$ \\
\hline 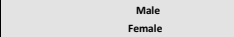 & $\begin{array}{l}63(59.2 \%) \\
56(47.1 \%)\end{array}$ & $\begin{array}{l}44(54.3 \% 8) \\
37.4588\end{array}$ & \\
\hline ASA score (1/2/3/4) & & & 0.11 \\
\hline 1 & $\begin{array}{l}65(52.1 \%) \\
42.3536)\end{array}$ & $\begin{array}{l}50(61.786) \\
253098 \%)\end{array}$ & \\
\hline${ }_{3}^{2}$ & $\begin{array}{l}42(35.3 \%) \\
14(11.8 \%)\end{array}$ & $\begin{array}{c}25(30.9 \%) \\
67740)\end{array}$ & \\
\hline 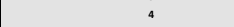 & $1(0.8 \%)$ & $0(0 \%)$ & \\
\hline $\begin{array}{l}\text { Tabagism } \\
\text { Type of surgery }\end{array}$ & $40(33.6 \%)$ & $27(33.3 \%)$ & $\begin{array}{l}0.99 \\
0.39\end{array}$ \\
\hline Orthopedics-Trauma & $78(66.786)$ & $63(77.8 \%)$ & \\
\hline Visceral (Digestive \& Gynecologic) & $29(24.8 \%)$ & $8(10 \%)$ & \\
\hline Head and Neck (incl. Neurosurgery) & $7(6 \%)$ & $5(6,2 \%)$ & \\
\hline \multirow{2}{*}{\multicolumn{4}{|c|}{ Aspiration \& full Stomach Risk Factors }} \\
\hline & & & \\
\hline GERD & $22(18.5 \%)$ & $7(8.6 \%)$ & 0.07 \\
\hline Heus & $13(10.9 \%)$ & $2(2.5 \%)$ & $0.03^{*}$ \\
\hline Morphine <4h before induction & $27(22.7 \%)$ & $25(30.9 \%)$ & 0.25 \\
\hline Chronic Kidney Disease & $7(5.98)$ & $2(2.5 \%)$ & 0.32 \\
\hline $\begin{array}{l}\text { Diabetes Mellitus } \\
\text { Nausea }\end{array}$ & 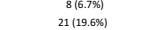 & $\begin{array}{c}6.7(7.48) \\
15(18.8 \%)\end{array}$ & $\begin{array}{l}0.99 \\
0.99\end{array}$ \\
\hline Vomiting & $13(12.1 \%)$ & $10(12.5 \%)$ & 0.99 \\
\hline \begin{tabular}{|l} 
Maximal Pain (Visual Analogicic Scale) \\
\end{tabular} & $6[5.4-7]$ & $7[5-7]$ & 0.98 \\
\hline $\begin{array}{l}\text { Maximal Stress (visual Analogic Scale) } \\
\text { Fasting Durations }\end{array}$ & $5[4.9-6]$ & $5[3-5]$ & 0.17 \\
\hline $\begin{array}{l}\text { Fasting Durations } \\
\text { From last liquid intake to induction }\end{array}$ & $11[10.2-12.4]$ & $12[10-14.3]$ & 0.49 \\
\hline From las solid intake to induction & $15[12.9-16.5]$ & $15.9[12.7-17.4]$ & 0.83 \\
\hline | Liquid intake ésh betore induction & ${ }_{11(9.65)}$ & $8(10 \%)$ & 0.99 \\
\hline 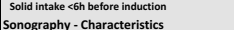 & & & \\
\hline $\begin{array}{l}\text { Ponography - Characteristics } \\
\text { Antral Cross Section Area (cm2) }\end{array}$ & $5.77(5.04-6.3]$ & $2.39[2.1 \cdot 2.6]$ & $<0.0001^{*}$ \\
\hline Ease of sonography (Visual Analogic Scale) & & $5[4-6]$ & $0.047^{*}$ \\
\hline Duration (min) & $5[4-5]$ & $5[5-5.9]$ & $0.03^{*}$ \\
\hline
\end{tabular}

Conclusion(s): Studying the second largest cohort described to date concerning gastric US in emergency anesthesia, clinical risk factors seem to fail at predicting full stomach and may lead to inadequate anesthetic strategies. Patients with clinically undetected full stomach do not present a specific risk factors pattern. Those data question clinical judgment regarding full stomach and plead for larger implementation of gastric US in research and practice.

\section{References:}

1. Bouvet L. et al. Anaesthesiology. 2011

2. Perlas A. et al. Anesth Analg. 2013 